\title{
Antiproton Driven Bi-Modal Fusion Propulsion System
}

\author{
T. Kammash* and R. Tang ${ }^{\dagger}$ \\ University of Michigan, Ann Arbor, MI, 48109
}

\begin{abstract}
The Gasdynamic Mirror (GDM) fusion reactor is investigated for utilization as a propulsion device, as well as for use as a surface power system when driven by antiprotons in both instances. The deuterium-tritium fusion reactions in the device will be initiated by the heating provided by the fission fragments and the annihilation products resulting from the "at rest" annihilation of antiprotons in $U^{238}$ target nuclei. The energetic pions and muons of the proton-antiproton (or neutron) annihilations in the $U^{238}$ nucleus can heat a DT plasma to several $\mathrm{keV}$ during their lifetime. The remaining heating to about $10 \mathrm{keV}$ is provided by the fission fragments. Fissioning of $U^{238}$ by "at rest" annihilation of antiprotons has been shown to be nearly $100 \%$ efficient, and the process can thus be effectively used in heating a suitable plasma to thermonuclear temperatures. With GDM as a steady state fusion reactor, and assuming certain efficiencies for the various components of the system, we calculate the energy multiplication factor " $Q$ " needed to sustain the steady state operation either in the "propulsive" mode or in the power-producing mode. With the aid of a system and mission analyses, we find that approximately 3.5 micrograms of antiprotons are required to accomplish a round trip mission to Mars in about 59 days. A similar amount is required to initiate and sustain the power-producing mode where gigawatts of electric power may be generated. Although roughly nanograms of antiprotons are currently produced annually, it is expected that hundreds of milligrams or even several grams will be produced annually in the next decade or so when Mars missions may be contemplated.
\end{abstract}

\section{Introduction}

A fusion reactor in which fusion reactions are triggered by fission fragments resulting from "at rest" annihilation of antiprotons in $\mathrm{U}^{238}$ targets is examined for propulsion and surface power applications. In the propulsion mode, a fraction of the escaping charged particles would be utilized to generate thrust while the remainder would be routed to a direct converter which, along with a thermal converter, will produce enough electric power as may be needed during the flight. As a surface power source the system can be used to perform whatever tasks it may be called upon to do. This may include, for example, mining Helium-3 on Jupiter whose atmosphere is known to contain $\mathrm{He}^{3}$ to the tune of 350 trillion tons compared to the much smaller amount in the lunar regolith. Since $\mathrm{He}^{3}$ is a desirable fuel for terrestrial fusion power reactors but virtually non-existent on Earth, and much more readily extractable from the atmosphere than from the solid regolith, we address in this paper a bi-modal fusion system that uses $\mathrm{D}-\mathrm{He}^{3}$ fuel cycle and assess its capability for transportation to and mining of a planet such as Jupiter. It has been estimated ${ }^{1}$ that one ton of $\mathrm{He}^{3}$ in combination with $2 / 3$ ton of deuterium burned in a fusion reactor at an efficiency of about $10 \%$ will produce about $1.85 \mathrm{GW}$ year of energy. If the annual global industrial energy consumption of $10^{4} \mathrm{GW}$ year is further assumed, then that is the equivalent of the use of 5400 tons of $\mathrm{He}^{3}$ in fusion reactors. Moreover, it has also been pointed out that the energy value of $\mathrm{He}^{3}$ in today's dollars is approximately $\$ 9 \times 10^{6}$ per kilogram when compared to the value and energy potential of oil. This suggests that securing $\mathrm{He}^{3}$ in large amounts even from distant planets such as Jupiter or Uranus might be economically acceptable. Another factor that may enter the consideration of mining the atmospheres of the Jovian planets is the amount of power required to extract $\mathrm{He}^{3}$. When transportation energy is excluded, it has been shown ${ }^{2}$ that it takes $84 \mathrm{GJ} / \mathrm{kg}$ for mining and $186 \mathrm{GJ} / \mathrm{kg}$ for gas separation for a total of $270 \mathrm{GJ}$ for mining one $\mathrm{kg}$ of $\mathrm{He}^{3}$ on the moon. This is to be contrasted with a total power of about 414 MW required for liquefaction and separation of $47.5 \mathrm{gm} / \mathrm{sec}$ in the atmosphere of Jupiter. ${ }^{3}$ This amounts to $8.7 \mathrm{GJ} / \mathrm{kg}$ or a factor of approximately 30 less than that of the moon case.

\footnotetext{
* Professor Emeritus, Dept. of Nuclear Engineering and Radiological Sciences, 2355 Bonisteel Blvd, Ann Arbor, MI 48109, AIAA Associate Fellow.

${ }^{\dagger}$ Graduate Student, Dept. of Aerospace Engineering, 1320 Beal Ave, Ann Arbor, MI 48109.
} 
The longer flights between these outer planets and the inner solar system may turn out to be less critical once a regular supply line had been set up. Certainly in the case of Uranus and Neptune, the lower escape velocities ( 20 $\mathrm{km} / \mathrm{sec}$ ) would greatly facilitate lifting the $\mathrm{He}^{3}$ collected off the planet and returning it to where it was required. The flight time to Jupiter for the proposed propulsion system will be presented in the next section. In the meantime, however, it may be interesting to note that Jupiter is only aligned correctly with Saturn once every 19.9 years, with Uranus once every 13.8 years and with Neptune once every 12.8 years. So it is quite reasonable for a space tanker, employing the proposed fusion propulsion system, to fly from earth to the outer planet of choice, spend a period of time in the planet's atmosphere extracting $\mathrm{He}^{3}$, or loading it from an extractor plant already in place, and then return to earth (or other destinations if need be) with its cargo.

\section{Fusion Propulsion System}

The propulsion component in the proposed system consists of a mirror type fusion reactor, specifically the Gasdynamic Mirror (GDM) wherein the plasma density and temperature are such that the ion-ion collision mean free path is much shorter than the plasma length. Under these conditions the plasma behaves much like a fluid, and its escape from the mirror is analogous to the flow of a gas into vacuum from a vessel with a hole. Fusion reactions are initiated indirectly by antiprotons stored in a trap that is connected to the GDM. Recent physics studies ${ }^{4}$ have shown that "at rest" annihilation of antiprotons in the uranium isotope $\mathrm{U}^{238}$ leads to fission at nearly $100 \%$ efficiency. The resulting highly charged, fast fission fragments are highly ionizing and can, along with the annihilation products namely the pions and muons, heat a fusion plasma to thermonuclear temperatures. In the system under consideration, antiprotons are released from the trap and allowed to strike the $\mathrm{U}^{238}$ target (in strip form) at a pre-determined position to trigger the desired nuclear reactions. A GDM device currently exists at NASA's Marshall Space Flight Center where experiments are being conducted on plasma confinement in such a magnetic geometry. An antiproton trap which may hold up to $10^{12}$ particles has also been designed and built at the same location to investigate the use of such particles in space propulsion.

Using D-He $\mathrm{H}^{3}$ fuel with and $\mathrm{He}^{3} / \mathrm{D}$ ratio of one and operating at $75 \mathrm{keV}$ temperature and a density of $10^{17} \mathrm{~cm}^{-3}$, we find that the neutron power is less than $5 \%$ of the fusion power, thus making the radiator mass much more acceptable than it would have been had a deuterium-tritium (DT) fuel cycle been employed. Assuming an injector efficiency of one, a thermal converter efficiency of 0.3 and a direct converter efficiency of 0.8 , we find that the Qvalue (ratio of fusion power to injected power) is about 1.56 which the GDM reactor is capable of delivering at the density and temperature noted above. It may be noted at this point that in spite of their relatively short lifetimes in the rocket frame (72 ns for the pions and $8.4 \mu$ s for the muons), the pions and muons contribute $5 \mathrm{keV}$ and $25 \mathrm{keV}$ respectively towards heating the plasma to the operating temperature of $75 \mathrm{keV}$. Moreover, we find that such a propulsion system will produce a specific impulse of $4.9 \times 10^{5}$ seconds at a thrust of $3.6 \times 10^{5}$ Newtons. When applied to a round trip mission to Jupiter using continuous burn, acceleration/deceleration type of trajectory, we find that the mission can be accomplished in about 359 days with the outbound trip taking approximately 178 days. A total of about 80 micrograms of antiprotons is required to initiate the fusion reactions and the subsequent steady state operation of the system. Although the total vehicle mass is quite large, the thrust power generated by the system, namely, $635 \mathrm{GW}$ yields a specific power $\alpha$ of about $19 \mathrm{~kW} / \mathrm{kg}$ and a thrust to weight ratio of $10^{-3}$ placing it at the higher end of the projected values for fusion propulsion systems. ${ }^{5}$ Clearly this $\alpha$ value is significantly higher than those projected for nuclear-electric, gaseous fission or nuclear thermal systems.

\section{Fusion Surface Power}

We noted above that large amounts of power are required to mind $\mathrm{He}^{3}$ from the atmosphere of Jupiter, and even larger amounts per kg are needed in the case of the moon. We recall that $414 \mathrm{MW}$ of power are needed to extract 47.5 grams per second of $\mathrm{He}^{3}$, and when the ascent energy of $114 \mathrm{MW}$ (Ref. 3) is added, we see that a total of 528 MW is needed to extract that amount and lift it into an orbit about Jupiter from which shipment to its destination may begin. Clearly, the proposed system in its "surface" power operating mode must be able to deliver that amount of power and more. Our calculation reveals that the system in question will generate $178 \mathrm{GW}$ of electric power when operating at a plasma density of $9 \times 10^{16} \mathrm{~cm}^{-3}$ and a temperature of $60 \mathrm{keV}$ with effectively the same efficiencies for the various components as in the propulsion mode. Approximately the same amount of antiprotons will be needed to start the system if, for one reason or another, the system was turned off upon arrival at Jupiter. These amounts of antiprotons far exceed the present annual production of nanograms, but significantly larger amounts, on the order of grams and possibly tens of grams, are expected to be achieved in the next decade or two ${ }^{6}$ to meet these needs. 


\section{Conclusion}

We have presented in this paper a bi-modal fusion system that can be used for transportation to, and the mining of $\mathrm{He}^{3}$ from, the Jovian planets. With the higher concentration of this isotope in the atmosphere of Jupiter $\left(\sim 10^{-4}\right)$ as compared to that in the lunar regolith $\left(10^{-8}-10^{-9}\right)$ (Ref. 7), and the significantly smaller power requirements for its extraction, it is shown that the proposed system would be capable of providing the needed power and the means of speedy transportation to supply the global needs for $\mathrm{He}^{3}$ for use in fusion power reactors. Moreover, it was demonstrated that modest amounts of antiprotons would be required to drive the system in either of the propulsion or surface power modes, and such amounts are projected to be available in the next couple of decades.

\section{References}

${ }^{1}$ Martin, A. R., and Bond, A., "Project Daedalus: The Propulsion System, Part 1 - Theoretical Considerations and Calculations,” Journal of the British Interplanetary Society, Supplement, 1978, pp. S57.

${ }^{2}$ Kulcinski, G. L., et al., "Energy Requirements for Helium-3 Mining Operations on the Moon," $5^{\text {th }}$ Symposium on Space Nuclear Power Systems, Albuquerque, NM, 1988, pp. 77.

${ }^{3}$ Parkinison, R. C., "Project Daedalus: Propellant Acquisition Techniques," Journal of the British Interplanetary Society, Supplement, 1978, pp. S83.

${ }^{4}$ Bocquet, J. P., et al., "Prompt Fission Induced by Antiproton Annihilation At Rest on Heavy Nuclei,” Z. Phys. A-Hadrons and Nuclei, Vol. 342, No. 2, 1992, pp. 183-189.

${ }^{5}$ Santarius, J. F., "Fusion Space Propulsion - A Shorter Time Frame Than You Think," $52^{\text {nd }}$ JANNAF Propulsion Meeting, Monterey, CA, 2005.

${ }^{6}$ Frisbee, R. H., "How to Build An Antimatter Rocket for Interstellar Missions," $39^{\text {th }}$ Joint Propulsion Conference, Huntsville, AL, 2003. Paper AIAA-2003-4676.

${ }^{7}$ Matloff, G. L., and Cassenti, B. N., “The Solar Wind: A Source for Helium-3,” $43^{\text {rd }}$ Congress of the International Astronautical Federation, Washington, D.C., 1992. 